\title{
Direct imaging of single Au atoms within GaAs nanowires
}

\author{
Maya Bar-Sadan $^{1,2^{\star}}$, Juri Barthel ${ }^{2}$, Hadas Shtrikman $^{3}$ and Lothar Houben ${ }^{2}$ \\ 1. Chemistry Department, Ben Gurion University of the Negev, Be'er Sheba, Israel \\ 2. Peter Grünberg Institut and Ernst Ruska-Centre for Microscopy and Spectroscopy with Electrons (ER-C), \\ Research Centre Jülich, 52425 Jülich, Germany \\ 3. Braun Center for Submicron Research, Weizmann Institute of Science, Rehovot 76100, Israel
}

*barsadan@bgu.ac.il

Keywords: Single atom detection, STEM

Incorporation of catalyst atoms during the growth process of semiconductor nanowires reduces the electron mean free path and degrades their electronic properties. Aberration-corrected scanning transmission electron microscopy (STEM) is now capable of directly imaging single Au atoms within the dense matrix of a GaAs crystal, by slightly tilting the GaAs lattice planes with respect to the incident electron beam. Au doping values in the order of $10_{17-18} \mathrm{~cm}_{3}$ were measured, making ballistic transport through the nanowires practically inaccessible.

Nanowires of III-V semiconductors are produced in specific compositions which enable the tuning of the nanowire's bandgap. In addition, these nanomaterials provide high electron mobility hence making them exceptionally interesting for photovoltaic devices as well as for other nano-electronic and nano-photonic devices. The control over unintentional incorporation of catalyst atoms during the growth is an important issue when producing high-quality wires for transport experiments. In particular, incorporation of atoms from the Au catalyst droplet is known to be a major problem in Si nanowires growth and is a source for debates concerning InAs and GaAs as well. It is an obstacle for transport experiments since it makes the ballistic transport practically inaccessible.

For these reasons, imaging of buried single dopant atoms is a long-sought goal. In general the HAADF signal intensity is enhanced with increasing atomic number producing chemically sensitive "Z-contrast". In a simplified but frequently applied approach the intensity of the HAADF-STEM signal is estimated to be proportional to $Z_{1.6-1.8}$. The difference between the atomic numbers $Z_{\text {host }}$ of the host lattice and $Z_{\text {dopant }}$ of the foreign atom enables the identification of dopant atoms within the matrix. Compared to previous works on systems with much larger $Z_{\text {dopant }} / Z_{\text {host }}$ ratios e.g. $Z_{\text {Au }} / Z_{\mathrm{si}}=5.6$, the detection of dopant atoms in the present case where $Z_{A u} / Z_{G a}=2.5$ can be considered as a very challenging task. A $Z$ ratio of 2.5 is mentioned in the literature to be insufficient for the imaging of single dopants within a matrix in zone-axis configurations.

However, estimating dopant detectability from the pure $Z_{\text {dopant }} / Z_{\text {host }}$ ratio neglects dynamical diffraction. In the usually applied zone-axis configuration, multiple elastic scattering events repeatedly focus the electron wave to the column. This so-called channelling effect enhances the wave amplitude at certain sites along the column resulting in a stronger high-angle scattering from the atoms in the sample column. However, the depths where the beam is re-focused depends for example on the chemical composition and on the distance between the atoms along the column. Therefore, the sensitivity for the detection of single dopant atoms in the zone-axis orientation is highly non-linear and depends on numerous material and instrument parameters.

Our approach is to apply a small tilt to the lattice planes relative to the incident beam direction. In this configuration the excess signal of $\mathrm{Au}$ atoms is enhanced over the background signal of adjacent $\mathrm{Ga}$ atoms since the high angle scattering from the column strongly decreases. We show the effect of such a small tilt on the detection sensitivity of a dopant atom by a simulation study of the elastic interaction between the electron probe and the atomic lattice using multislice calculations.

Two important observations from the simulations are: (i) With a tilted sample, a very strong SE is obtained when the Au atom is close to the focus plane of the electron probe. (ii) The detection of $\mathrm{Au}$ atoms with a Sub-Ångstrom probe stems from a fraction of the depth of the sample when using single focus images.

Dopant atoms are detected in Fig. 1 as intensity peaks against the background signal from the GaAs matrix. The horizontally and vertically striped lattice images of GaAs depict the intentional tilts of the nanowire relative to the beam direction. The detection limit of the SE in the experimental images is determined essentially by the noise level, which depends on the electron dose and on the 
noise of the background intensity of the GaAs matrix. In addition, thin surface layers of gallium oxide and hydrocarbon contamination lower the SE due to beam broadening and contribute to the noise in the experiment. The peak maximum reaches a signal enhancement of up to $25 \%$, which is well beyond the experimental noise as marked by the gray bands. We find significant intensity peaks above the background noise, which we associate with $\mathrm{Au}$ atoms. The calculated density of the Au atoms is between 0.025 and 0.1 per image area of $1 \mathrm{~nm}_{2}$. From the simulations we approximate that the generation of a significant signal from the Au atoms is restricted to a depth range of about $3 \mathrm{~nm}$ as an upper limit. Therefore the lower limit of the doping level is estimated by $10 \mathrm{ppm}$ or $10_{17} \mathrm{~cm}_{-3}$ for a 10-15 nm thick nanowire. Such a doping level is close to the maximum solubility of $\mathrm{Au}$ in GaAs, which can reach values in the order of $10_{16-18} \mathrm{~cm}-3$. Therefore, the incorporation of the gold atoms within the GaAs nanowires is high, possibly even reaching supersaturation, as reported previously for InAs nanowires. Hence GaAs NWs synthesized by the VLS method do not provide the required impurity level for ballistic transport studies, and a self-catalytic process may be a favorable synthetic route.

\section{References}

[1] M. Bar-Sadan, J. Barthel, H. Shtrikman, L. Houben, Nano.Lett., 12, 5, 2352-2356 (2012).
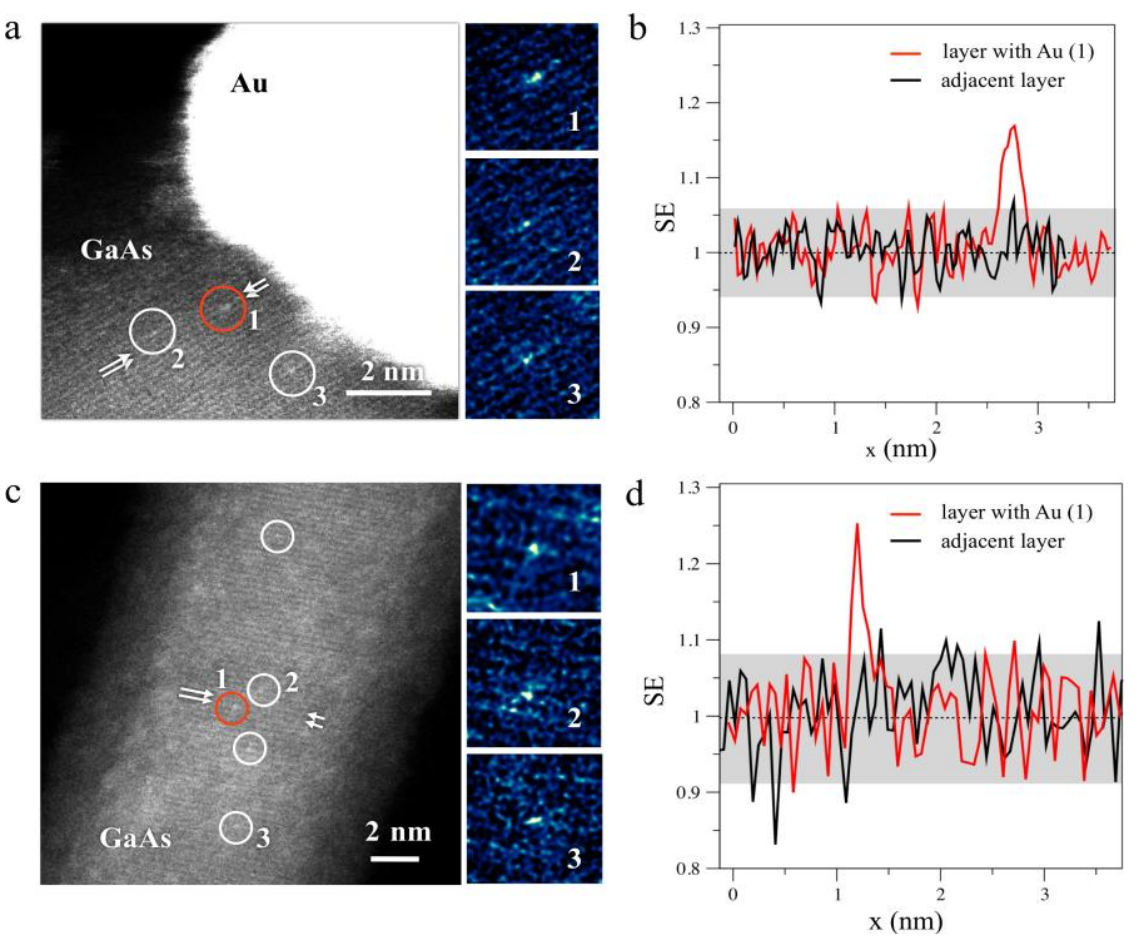

Figure 1. Experimental HAADF images (a) and (c) and SE line profiles (b) and (d) taken along lattice planes of a GaAs nanowire in a projection slightly off a zone axis. The circles in the HAADF images mark intense peaks with a SE well above the experimental noise level. In each image, three of these peaks were selected, and displayed enlarged in the false color insets to the right. For the peaks marked by 1 and a red circle, intensity line profiles along lattice planes are presented in (b) and (d). The red curve in the profiles is the SE along a lattice plane containing an intense peak, which is attributed to a single Au atom. In comparison, the black curves show the SE along a neighboring lattice plane containing no Au atom. The estimated experimental noise levels are denoted by the grey bands. 